\title{
Application Géomatique Pour La Cartographie De La Vulnérabilité Environnementale Engendrée Par Les Déchets Miniers De La Mine Ferrifere De Nador (Nord-est du Maroc)
}

\author{
Mohamed Lakrim \\ Laila Mesrar \\ Omar El Aroussi \\ Raouf Jabrane
}

Faculté des sciences et techniques de Fès, Route d’Imouzzer. Maroc

doi: 10.19044/esj.2016.v12n15p287 URL:http://dx.doi.org/10.19044/esj.2016.v12n15p287

\begin{abstract}
The field work has been done to study the impact of acid mine drainage on the environment. Space pollution measurement was carried out on the basis of interesting physicochemical and geomatic analyses of water and solid region outcrops. The study showed that the phenomenon under study represents an alarming problem that is attributed to the presence of huge stocks of mining waste sulphur, directly exposed to a Mediterranean climate and thus remains capable of accelerating the oxidation process. The classification of zones according to the levels of fragility is entailed by the necessity to opt for a model that is compatible with different intrinsic and extrinsic parameters. The study was concluded by the superposition of six thematic layers charting the environmental vulnerability map by the GIS. The findings demonstrated that most vulnerable zones near the points of exploitation are very oxidized in bottomlands or in places well apart from carbonate facies $(\mathrm{pH}<3$.
\end{abstract}

Keywords: Acid mine drainage, mining waste sulphur, oxidation, environmental vulnerability, GIS

\section{Résumé}

Un ensemble de travaux sont réalisés afin d'étudier l'impact du drainage minier acide sur l'environnement. L'évaluation spatiale de la pollution a été réalisée à base des analyses physico-chimiques et géomatiques intéressantes les eaux autant que les afleurements solides dans la région. Le phénomène est identifié comme un problème alarmant, 
occasionné par la présence d'énormes stocks de déchets miniers sulfurés, exposés directement à un climat méditerranéen, capable d’accélérer le processus d'oxydation. La classification zonale en fonction de la fragilité est basée sur le choix d'un modèle compatible aux différents paramètres intrinsèques et extrinsèques. L’étude s’est achevée par la superposition de six couches thématiques et le traçage de la carte de la vulnérabilité environnementale en moyennant le SIG. Les zones les plus vulnérables sont constatées très oxydées $(\mathrm{pH}<3)$, localisées au voisinage des points d'exploitation et bien écartées des endroits sommitaux ou carbonatés.

Mots-clés: Drainage minier acide, déchets miniers sulfurés, oxydation, vulnérabilité environnementale, SIG.

\section{INTRODUCTION:}

Les gisements ferrifères du Maroc Oriental sont situés au sud-ouest de la ville de Nador. Les exploitations s'étant sur une superficie de $36 \mathrm{Km}^{2}$ d'un terrain assez irrégulier, composé d'un ensemble de collines tranchant topographiquement sur les plaines avoisinantes, où l'altitude s'élève jusqu' au 696m à Jbel Ouiksan, le point culminant du massif du massif des Béni Bou Ifrour (El Mansouri, 1985). Le secteur minier comprend cinq principales carrières d'exploitation à savoir; Ouiksan, Axara, Imnassen, Bokkoya et Setolazar (Fig. 1).

Le présent travail a pour objet de procéder à une synthèse des différents résultats obtenus afin d’évaluer les retombés environnementale réelles relative à chaque type de rejet minier, de le classer conformément à l'intensité du risque qu'il puisse causer à l'environnement et par conséquent à l’homme. Le concept du risque pris en compte dans cette étude est celui du risque généré par le drainage minier acide (DMA). Les polluants issus des exploitations minières et activités connexes peuvent être transférés, pour contaminer les compartiments environnementaux liquides et solides. (Lakrim et al, 2012).

La classification des rejets miniers est définie par rapport à l'ampleur de risque, de manière que la répartition spatiale des risques est en fonction de l'importance des paramètres intrinsèques et extrinsèques appréhendés. La cartographie de la vulnérabilité environnementale se traduit alors en probabilité d'occurrence d'un problème sanitaire et/ou environnemental lié à la combinaison de plusieurs paramètres physiques susceptibles de contribuer à la contamination de la population pendant une période donnée suite à leur exposition aux flux métalliques (incident potentiel). Pour chaque type de rejets minier rencontré, le risque est exprimé en fonction des propriétés intrinsèques génératrices du DMA, et de quelques caractéristiques extrinsèques (la nature des pentes à titre d'exemple). 


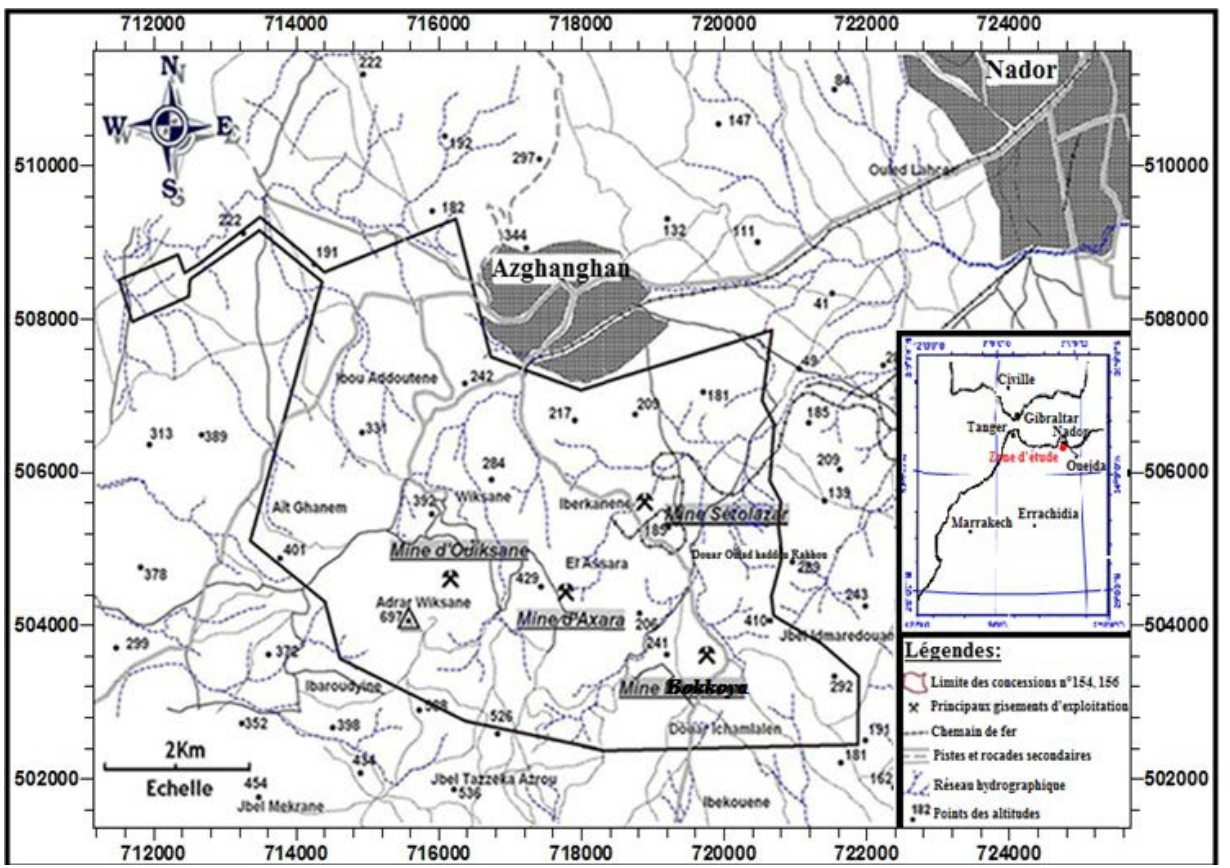

Fig. 1 : Position géographique des concessions minières et des gisements ferrifère dans la zone d'étude.

\section{MATÉRIELS \& MÉTHODES}

\section{II.1. Échantillonnage}

L'échantillonnage concerne les compartiments solides (sols, déchets miniers...) et liquides de différents niveaux disponibles dans le site minier. Il concerne exceptionnellement les tranches superficielles allant de 30 à $50 \mathrm{~cm}$ de profondeur. Le choix des échantillons s'est fait selon la méthode de prélèvement ponctuel aléatoire, de sorte que les échantillons soient rigoureusement représentatifs de toutes les unités constituant les formations apparentes ou accessibles, en garantissant toutes les caractéristiques de fond et de forme. Par ailleurs, plus de 100 échantillons, d'un poids exhaustif total de $90 \mathrm{~kg}$, provenant des sites miniers et des alentours. Ils ont été prélevés directement des aires d'entreposage, de quelques champs, des cours d'eau, des puits, des galeries ...etc. L'échantillonnage concerne les différentes périodes de l'année, depuis 2007 jusqu'à 2014. À l'échelle macroscopique et minéralogique, les échantillons sont aperçus très hétérogènes avec la prédominance des concentrations excessives en fer et en soufre.

\section{II.2. Méthodes analytiques}

L'évaluation des retombés significatives des rejets miniers sur l'environnement, dépend des méthodes analytiques inspirées à partir des 
essais expérimentaux qualifiés pour mettre en considération la quantité des déchets rejetée, ainsi que la surface occupée et leur teneur en éléments métalliques pouvant être libérés dans les écosystèmes. Le classement spatial des zones fragiles s’effectue selon l’intensité du risque de DMA, généré par les rejets miniers stockés à l'air libre, exhibés aux conditions intrinsèques et extrinsèques diversifiées. La connaissance des propriétés intrinsèques des déchets miniers est indispensable. À titre d'exemple, deux rejets ayant un même taux de lixiviation métallique, mais des concentrations différentes ne présentent pas le même risque par rapport à l'environnement (Kaniki, 2008). Le taux de lixiviation et la teneur doivent être combinés pour obtenir un nouveau paramètre susceptible d'évaluer les risques que ces rejets présentent pour l'environnement. Ce nouveau paramètre peut même contribuer à différencier le classement du rejet minier. Les autres paramètres sont différenciés selon le pouvoir susceptible de produire ou de contribuer à la génération des polluants. L’élaboration des cartes thématiques est basée sur le concept de classement sous forme d'intervalles de valeurs numériques, correspondantes à chaque niveau. Ce rangement dépend de la susceptibilité de produire ou d'accélérer le processus de DMA. L’approche vise à bien façonner une carte de la vulnérabilité environnementale, comme résultat de la combinaison par superposition des différents paramètres physiques reportés en cartes thématiques. L'application des systèmes d'information géographique (SIG) s’avère adéquate. La carte des risques a été réalisée à partir de la superposition des diverses cartes thématiques incorporant la répartition des rejets miniers, la répartition des pentes, le potentiel d’hydrogène $(\mathrm{pH})$, la conductivité électrique, la répartition géochimique du fer et du soufre.

\section{RÉSULTATS \& DISCUSSIONS \\ III.1. Indexation des différents facteurs}

La cartographie des risques de contaminations est basée sur la définition des facteurs majeurs générateurs du DMA, comme une probabilité d’occurrence sur la zone minière. La méthodologie vise à choisir les zones les plus touchées et prioritaires pour l'aménagement, en s’appuyant sur des analyses qualitatives et quantitatives des facteurs à l'origine de la génération et de l'activation du DMA. L'analyse des mécanismes de chaque entité inventoriée nous a permis de dégager le degré de contamination et de sélectionner l'ampleur du risque existant ou susceptible d'être généré (Lakrim, 2015).

Les facteurs de fragilité sont classés qualitativement de bon, moyen ou mauvais, par la qualité de l'indice retenue. On a ensuite cherché à évaluer l'importance de chacun des facteurs gradués de manière simple, arithmétique, en les classant suivant une échelle numérique au nombre de 
cinq. Les zones ainsi obtenues sont codées, généralement de 0 à 4 selon l'indice de qualité (Tab.1). La méthode s'applique en mode «raster», c'est à dire, la zone d'étude est subdivisée en grille constituée de petites zones appelées cellules, dont la forme est carrée et dans laquelle les différents paramètres sont homogènes (Lakrim, 2015). L’analyse des facteurs permanents et les seuils qu'ils présentent dans les zones reconnues fragiles ont conduit à définir des zones de présomptions (Margaa, 1994). Nous allons passer en revue les facteurs qui sont déterminants dans l'évaluation des potentialités de contamination du sol et de l'eau dans le site.

Tab. 1 : Indices de classement selon l'intensité des contaminations.

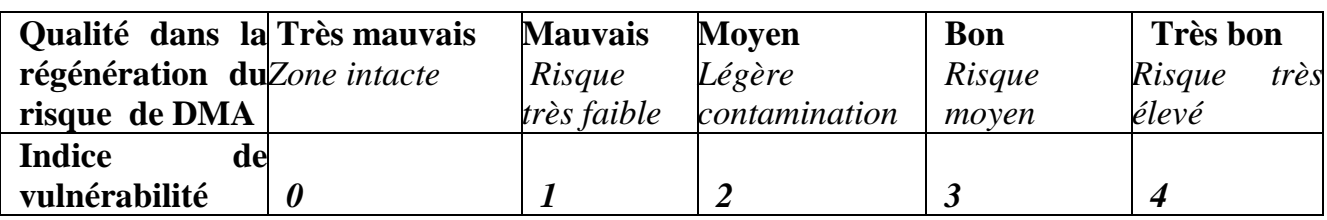

\section{III.2. Élaboration des cartes thématiques}

\section{III.2.1. Carte des rejets miniers}

Les résultats des travaux antérieurs permettent de conclure que les faibles quantités métalliques mobilisables sont enregistrées par les remblais, en revanche les plus fortes quantités sont marquées clairement par les terrils et les tailings de la digue à stériles de Sétolazar (Lakrim, 2015). La quantité mobilisée est liée à la nature de rejet, à la teneur totale en l'élément métallique toxique, à la granulométrie et surtout à la teneur en $\mathrm{S}$ et en Fe qui sont responsables de l'abaissement des $\mathrm{pH}$ des milieux (production de $\mathrm{H}^{+}$et $\mathrm{H}_{2} \mathrm{SO}_{4}$ ). Ces résultats permettent le classement suivant en fonction de l'importance du risque de libération des éléments métalliques:

Tailings $>$ Terrils de Sétolazar $>$ Terrils d'Ouiksane $>$ Terrils d'Axra > Remblais d'Ouiksane $>$ Remblais de Sétolazar

Pour confirmer la classification des rejets miniers rencontrés sur le terrain, on évoque d'autres critères proposés utiles qui peuvent contribuer à collationner les risques environnementaux pour traduire une logique d'ordre. Les risques qui peuvent être régénéré par un stock de déchet minier mis en décharge en plein air et dépendent asservissement de plusieurs paramètres. Les plus importants qui ont été déterminés sont le taux de lixiviation, la teneur en polluants métalliques et la perméabilité. La toxicité relative de chaque polluant métallique a été appréciée comme effets néfastes sur l'homme ou dévastateurs sur l'écosystème. L'ensemble des paramètres ont été traités dans les travaux antérieurs afin de classer les rejets miniers reportés sur carte selon le degré de contamination (Lakrim, 2015). Le principe de la toxicologie des éléments métalliques a permis de mettre en ordre un classement qualitatif des polluants $(\mathrm{Cu}, \mathrm{Zn}$, Ni et $\mathrm{Pb}$ ), selon l'ampleur de toxicité (Kaniki, 2008). En outre, le Zn est comme moins nocif 
que comparée aux Cu et Ni. De même, le nickel est considéré plus nocif que le cuivre. Le $\mathrm{Pb}$ n'a aucune fonction biologique dans les organismes vivants et sa toxicité commence à s'établir même à de très faibles teneurs (Chumbley, and Unwin, 2004). En plus, il a un caractère persistant et peut durer assez longtemps pour être s'éliminer de l'organisme (Baize, and Terce, 2002). Ces conclusions peuvent permettre de classer les rejets miniers à quantités mobilisables égales, en fonction de l'intensité de risque produit en ordre décroissant comme suite $\mathrm{Pb}>\mathrm{Ni}>\mathrm{Cu}>\mathrm{Zn}$.

Le classement des déchets miniers a nécessité d'intégrer, trois paramètres quantitatifs définis par le taux de lixiviation, la teneur et la perméabilité, plus un quatrième qualitatif qui caractérise la toxicité. Ces données ont été passées par un traitement articulé sur des méthodes élaborées à base d'analyses statistiques multivariée ou multicritère.

L'analyse multicritère (AMC) est un outil d'aide à la décision développé pour résoudre des problèmes multicritères complexes qui incluent des aspects qualitatifs et/ou quantitatifs dans un processus décisionnel (Renaud, in Kaniki, 2008). La procédure de classement à base d'analyse AMC, doit satisfaire la mise à l'échelle ou normalisation des indicateurs, l'établissement des matrices des éléments de décision simple, le traitement des valeurs numériques et le classement des alternatives (Renaud, 2006; Mendoza \& Macoun, 2000). Cependant, l'identification des indicateurs et la détermination des critères visent à intégrer quatre indicateurs : le taux de lixiviation (L), la concentration (C), la perméabilité (K) et la toxicité (T) (Kaniki, 2008). La procédure d'analyse multicritère consiste à employer la fonction cible (E.1):

$R=E i * f i$

$\boldsymbol{R}:$ risques (fonction cible) qui associe à chaque type de rejets $\boldsymbol{E} \boldsymbol{i}$ (éléments) un critère $\boldsymbol{f i}$ défini par la relation susmentionnée. Le critère $\boldsymbol{f i}$ est un indicateur ou une combinaison d'indicateurs. Le nombre d'indicateurs n, étant égal à 4, la relation (E.2) ultérieure permet de déterminer le nombre total de critères de la manière suivante :

$f_{j}=\sum_{i=1}^{n} C_{n}^{i} \quad$ avec $\quad C_{n}^{i}=\frac{n !}{i !(n-i) !}$ donc $f_{j}=\sum_{i=1}^{4} C_{4}^{i}=\left(C_{4}^{1}+C_{4}^{2}+C_{4}^{3}+C_{4}^{4}\right)=15$

Connaissant le nombre d'échantillons $\boldsymbol{E}_{\boldsymbol{i}}$ et le nombre de critères $\boldsymbol{f}_{\boldsymbol{j}}$ on peut écrire la fonction cible (E.1) sous forme de la matrice d'évaluation (E.3) (Kaniki, 2008). Il ya 15 critères en question, employée afin d'évaluer les risques que peut présenter chaque rejet. Ces critères sont : $L, C, T, K, L C, L T$, $L K, C T, C K, T K, L C T, L C K, C T K, L T K$ et LCTK. L'intensité du risque s'est articulée sur le critère L.C.T.K qui associe tous les indicateurs comme étant approprié sous réserve du poids en tenant compte de la pondération de 
chaque indicateur dans le critère. Les autres éléments de décision peuvent être considérés comme des cas particuliers du critère $L C T K$.

$$
R=E_{i} * f_{j}=\left(\begin{array}{ccccc}
f_{1}(\text { T.o }) & f_{1}(\text { R.o }) & \ldots & \ldots & f_{1}(T L N) \\
f_{2}(\text { T.o }) & f_{2}(\text { R.o }) & \ldots & \ldots & f_{2}(T L N) \\
f_{3}(\text { T.o }) & f_{3}(\text { R.o }) & \ldots & \ldots & f_{3}(T L N) \\
\ldots & \ldots & \ldots & \ldots & \ldots \\
\ldots & \ldots & \ldots & \ldots & \ldots \\
\ldots & \ldots & \ldots & \ldots & \ldots \\
f_{15}(\text { T.o }) & f_{15}(\text { R.o }) & \ldots & \ldots & f_{15}(T L N)
\end{array}\right)
$$

L'indexation des variables ou bien la mise à l'échelle des différentes variables indicateurs de la pollution s'est effectuée par la normalisation des unités, pour but de les rendre homogènes et susceptibles d'être traitables numériquement. Ces indicateurs seront traités en fonction de leurs qualités et/ou quantités. Nous avons choisi alors, d'attribuer à chaque indicateur quatre valeurs comme l'indique le tableau 2 et la qualification de chacune d'elle est présentée au tableau 3.

Tab.2 : Tableau d'indexation des variables indicateurs d'intensités des risques croissants.

\begin{tabular}{|l|l|l|l|l|}
\hline \multirow{2}{*}{ Indices } & Valeurs & $\boldsymbol{C}$ & $\boldsymbol{T}$ & $\boldsymbol{K}$ \\
\cline { 2 - 5 } $\mathbf{L}$ & $\mathbf{L}$ & ] $0 \% ; 1 \%[$ & $\mathrm{Zn}$ & $<1.10^{-8}$ \\
\hline $\mathbf{2}$ & ] $1 \% ; 10 \%[$ & ] $0.05 \% ; 0.5 \%[$ & $\mathrm{Cu}$ & ] $1.10^{-8} ; 1.10^{-7}[$ \\
\hline $\mathbf{3}$ & ] $10 \% ; 50 \%[$ & ] $0.5 \% ; 1 \%[$ & $\mathrm{Ni}$ & ] $1.10^{-7} ; 1.10^{-5}[$ \\
\hline $\mathbf{4}$ & $>50 \%$ & $>1 \%$ & $\mathrm{~Pb}$ & $>1.10^{-4}$ \\
\hline
\end{tabular}

Tab. 3 : Tableau de qualification des paramètres indicateurs de la pollution.

\begin{tabular}{|c|c|c|c|c|}
\hline \multirow[b]{2}{*}{ Indices } & \multicolumn{4}{|l|}{ Qualification } \\
\hline & $\begin{array}{l}\text { Lixiviabilité } \\
\text { «L» }\end{array}$ & $\begin{array}{l}\text { Concentration } \\
« C »\end{array}$ & $\begin{array}{l}\text { Toxicité } \\
« T »\end{array}$ & $\begin{array}{l}\text { Perméabilité } \\
\text { «K»}\end{array}$ \\
\hline 1 & $\begin{array}{l}\text { Peu Lixiviable } \\
L=1\end{array}$ & $\begin{array}{l}\text { Pauvre } \\
C=1\end{array}$ & $\begin{array}{l}\text { Peu toxique } \\
T=1\end{array}$ & $\begin{array}{l}\text { Peu perméable } \\
K=1\end{array}$ \\
\hline 2 & $\begin{array}{l}\text { Lixiviabilité modérée } \\
L=2\end{array}$ & $\begin{array}{l}\text { Peu concentré } \\
=2\end{array}$ & $\begin{array}{l}\text { Toxicité modérée } \\
T=2\end{array}$ & $\begin{array}{l}\text { Assez perméable } \\
K=2\end{array}$ \\
\hline 3 & $\begin{array}{l}\text { Lixiviable } \\
L=3\end{array}$ & $\begin{array}{l}\text { Assez concentré } \\
C=3\end{array}$ & $\begin{array}{l}\text { Toxique } \\
T=3\end{array}$ & $\begin{array}{l}\text { Perméable } \\
K=3\end{array}$ \\
\hline 4 & $\begin{array}{l}\text { Très Lixiviable } \\
L=4\end{array}$ & $\begin{array}{l}\text { Concentré } \\
C=4\end{array}$ & $\begin{array}{l}\text { Très toxique } \\
T=4\end{array}$ & $\begin{array}{l}\text { Très perméable } \\
K=4\end{array}$ \\
\hline
\end{tabular}

L’établissement des matrices des éléments de décision simple est la phase de la mise en ordre des paramètres intéressants le taux de lixiviation, la teneur, la toxicité et de la perméabilité, selon une échelle qui concède 
l'élaboration des matrices élémentaires. La dernière étape pour rendre la comparaison simple, afin de faciliter le traitement numérique des données indexées, on attribue également à la fonction cible quatre valeurs correspondant aux qualifications : rejets à faible risque ; rejets à risque modéré, rejets à risque assez élevé et rejets à risque élevé.

La définition des indicateurs se succède suivant une opération de multiplication «*» fondée sur la qualité attribuée à chaque indicateur. Elle permet de fournir à la fonction objectif quatre valeurs correspondant aux qualifications précitées. Nous définissons donc, l’opération produit «*» ayant les propriétés suivantes :

Indice 1: le produit de deux valeurs identiques donne un résultat identique. Exemple d'un rejet peu lixiviable et renferme un polluant peu toxique (niveaux: 1 et 1), le rejet est classé à faible risque.

Indice 2: le produit de la plus faible valeur à la valeur la plus élevée donne une valeur intermédiaire. Exemple d'un rejet peu lixiviable et renferme un polluant toxique (niveaux: 1 et 4), le rejet est classé à risque non négligeable.

Indice 3: le produit d'une valeur inférieure à une valeur supérieure donne une valeur supérieure à la valeur inférieure. Exemple d'un rejet peu lixiviable contenant un polluant très toxique, le rejet est à risques modérés.

Indice 4: l'opération produit est commutative, c'est-à-dire que le résultat est le même, quel que soit l'ordre des facteurs. Le rejet à risque fort.

Le calcul numérique en fonction des indices attribués à chacun des critères, en s'appuyant sur la méthode susmentionnée et citée par Kaniki (2008)., on peut conclure le résultat traduit par la solution de la convergence matricielle (résultats des analyses géochimiques des travaux antérieurs in Lakrim, 2015), comme suite:

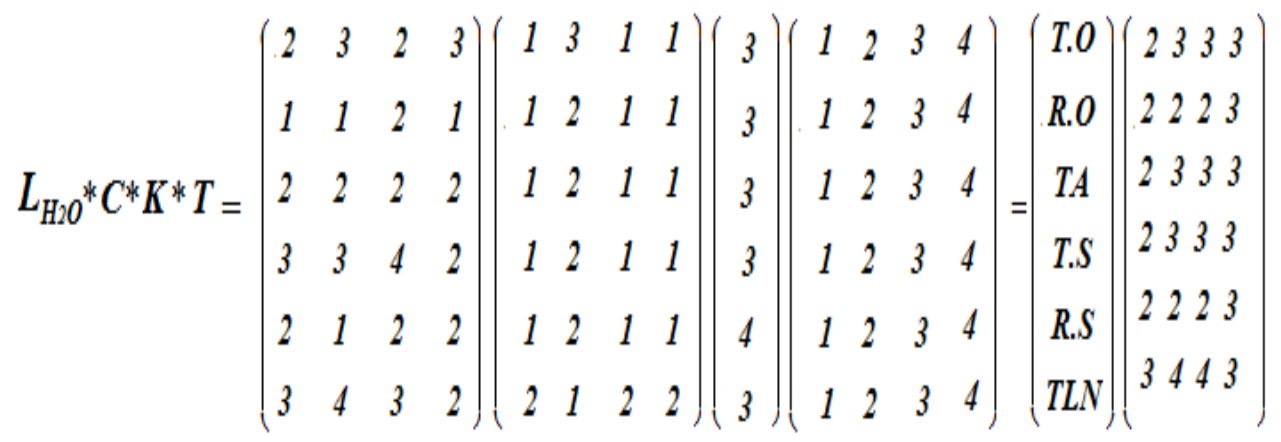

À travers cette solution on peut déduire l'intensité du risque suivant la méthode d'analyse multicritère selon une échelle à risque décroissante : TLN $>$ T.S $>$ T.A $>$ T.O $>$ R.O $>$ R.S.

Le même principe appliqué pour la classification des rejets miniers répartis dans l'air d'étude afin de sélectionner les zones les plus vulnérables 
et prioritaires pour l'aménagement. Les tailings (TLN) de la digue ou ceux qui sont rassemblés ou disséminés à proximité de l'usine de traitement sont classés les plus dangereux, générateurs de DMA et de métaux toxiques. Le deuxième rang sera occupé par les terrils et notamment ceux de Sétolazar où le $\mathrm{pH}$ est le plus bas $(\mathrm{pH}=0.87(2010))$. Ensuite, les terrils d'Axara et ceux d’Ouiksan. Enfin, le dernier rang est réservé aux remblais, précisément les remblais d'Ouiksan, de Sétolazar et ceux de Bokkoya. Les rejets miniers sont classés selon les critères définis précédemment dans les essais d'évaluation des risques. Ils sont aussi classés selon leurs natures, la couleur caractérisant l'ampleur d'oxydation et selon l'existence des eaux acides. On distingue dès lors cinq classes qui sont codifiées de 0 à 4 (Fig.2).

Il est important de noter que les quatre paramètres retenus ne sont pas les seuls paramètres indicateurs des risques. Il y a bien d'autres paramètres qui seront additionnés sous forme des cartes thématiques en couches d'informations, tels que l'emplacement par rapport à la pente, le $\mathrm{pH}$ des milieux, la quantité totale des déchets sur le site, la nature du substratum et la répartition géochimique des Fe et $\mathrm{S}$ qui influencent profondément le DMA.

\section{III.2.2. Carte de la répartition des pentes}

Les pentes sont classées en quatre catégories, indexées ou codifiées de 1 à 4 selon la possibilité de contribuer à la génération du DMA (Fig. 3). On peut donc, définir les zones à intensité de risque croissant comme suit :

Indice 1: Zone de très faible risque, dont la pente dépasse $25 \%$, où les conditions sont défavorables pour la stagnation des eaux et l'oxydation des minéraux sulfureux.

Indice 2 : Zone à risque moyen, dont la pente varie entre $15 \%$ et 25\%. Ces pentes favorisent légèrement le processus de DMA en présence de la matière sulfureuse, de l'air et des gouttelettes d'eau suspendues entre les débris.

Indice 3: Zone à risque fort, dont la pente varie entre 5\% et 15\%. Ces pentes favorisent une circulation ralentie des eaux pluviales et permettent l'engendrement du processus de DMA.

Indice 4 : Zone à très fort risque, dont la pente est inférieure à 5\%. Cette faible pente favorise la stagnation des eaux pluviales. Par conséquent, la combinaison de ces eaux avec les matériaux sulfureux (pyrite, chalcopyrite, pyrrhotite,...) en présence d'oxygène, peut conduire à des réactions d'oxydation des sulfures (E1) comme ci-après :

$\mathrm{FeS}_{2}(\mathrm{~s})+7 / 2 \mathrm{O}_{2}(\mathrm{~g})+\mathrm{H}_{2} \mathrm{O}(\mathrm{l})==>\mathrm{Fe}^{+2}(\mathrm{aq})+2 \mathrm{SO}_{4}{ }^{2-}(\mathrm{aq})+2 \mathrm{H}^{+}(\mathrm{aq})+$ énergie

\section{III.2.3. Carte de la répartition géochimique des sulfures}

La présence d’une quantité appréciable des minéraux sulfurés (pyrite, chalcopyrite, covellite, pyrrhotite, sphalérite et galène) dans les déchets 
miniers peut influencer positivement l'oxydation des composés sulfurés en présence des agents météoritiques (Lakrim, 2015). Ils peuvent provoquer ainsi l'abaissement des $\mathrm{pH}$ des milieux environnants, selon plusieurs réactions d'oxydation conformément à l'équation (E2) suivante : Minéral sulfureux $+\mathrm{O}_{2}+\mathrm{H}_{2} \mathrm{O}===>$ sulfate + acidité + métaux

Sur la base de la quantité du soufre présentée dans les rejets miniers, nous avons distingué cinq zones à caractères distinctifs (Fig. 4). Elles sont codifiées de 0 à 4 selon les normes limites suivantes :

Indice 0: Zone intacte, dont la concentration en soufre est strictement inférieure à $350 \mathrm{ppm}$. C'est la teneur mesurée dans l'eau de robinet admissible par le service de distribution de l'eau potable, destinée à la consommation de la population de la ville d'Azghanghan.

Indice 1 : Zone sensible, dont la teneur varie entre $350 \mathrm{ppm}$ et 450 $\mathrm{ppm}$. Cet intervalle définit la concentration en soufre dans l'eau de robinet de la ville d'Azghanghan et la teneur en soufre dans le puits situé à Douar Ichamlalene, qui approvisionne la population locale en eau, sous contrôle de l'office national de l'eau potable (ONEP) en 2011.

Indice 2 : Zone critique à risque faible, dont la teneur est entre 450 ppm et $500 \mathrm{ppm}$. Cet intervalle définit la teneur dans le puits sis à Douar Ichamlalene et la norme admissible par l'Organisation Mondiale de la Santé (OMS) pour les eaux de surface admissibles pour produire de l'eau potable.

Indice 3 : Zone à risque moyen, dont la teneur est entre $500 \mathrm{ppm}$ et $4700 \mathrm{ppm}$. Cet intervalle est défini la marge limite admissible par l'OMS pour les eaux de surface et la teneur en soufre existant dans l'un des puits abandonnés sis à Sétolazar.

Indice 4 : Zone à risque fort, dont la teneur est supérieure à 4700 $\mathrm{ppm}$, indiquant les zones riches en sulfures, là où la contamination est marquée plus avancée et s'intensifiée proportionnellement à l'acidité des milieux.

\section{III.2.4. Carte de la répartition géochimique du fer}

La répartition du fer avec des teneurs considérables dans les zones d'oxydation en présence d'un faible $\mathrm{pH}$, inférieur à 3 , peut importer beaucoup sur la cinétique des réactions d'oxydation. La présence du fer ferrique dans le milieu acide peut conduire à l'oxydation des minéraux sulfurés, même en absence totale d'oxygène. Pour cette raison, la cartographie de la répartition du fer s'en rend indispensable pour la définition de la vulnérabilité dans la zone.

La carte de la répartition du fer a également été subdivisée en cinq classes, indexées selon les niveaux des contaminations (Fig. 5). On spécifie donc les catégories suivantes : 
Indice 0 : Zone intacte, dont la teneur en fer est inférieure à 5 ppm. C'est la teneur minimale admissible par l'Organisation Mondiale de la Santé (OMS) pour les eaux de surface.

Indice 1 : Zone normale, dont la teneur varie entre 5 ppm et 50 ppm. C'est l'intervalle admissible par l'OMS pour les eaux de surface.

Indice 2 : Zone contaminée, dont la teneur varie entre 50ppm et 210ppm. Cet intervalle définit la norme admissible par l'OMS pour les eaux de surface et la norme marocaine admissible des eaux de surface.

Indice 3 : Zone à risque, dont la teneur varie entre 210 ppm et 2100 ppm. Cet intervalle définit la norme marocaine admissible pour les eaux de surface et la teneur mesurée dans quelques puits abandonnés sis à Sétolazar.

Indice 4 : Zone à risque fort, dont la teneur est strictement supérieure à 2100 ppm. Ces endroits sont caractérisés par la présence des eaux acides à faible $\mathrm{pH}$ où l'oxydation est intense.

\section{III.2.5. Carte de la répartition du pH des eaux}

Au-delà des modifications physiques que subissent les matériaux exposés aux différents agents climatiques, le phénomène de DMA progresse considérablement, en rendant les surfaces liquides plus acidifiées. Le pH des milieux peut importer beaucoup sur la cinétique des réactions d’oxydation des sulfures. La pyrite est le minéral le plus fréquemment rencontré dans les déchets, dans les parois des galeries et des carrières de la mine. L’oxydation des déchets miniers sulfurés ou pyriteux s'est y déroulé en deux étapes liées asservissement de la nature du $\mathrm{pH}$-milieu. On peut assister une oxydation directe et une autre indirecte. Le classement des intervalles d'intensités de génération du DMA est influencé par le potentiel d’hydrogène, ces résultats sont reportés dans la carte, illustrée dans la figure. 6:

Indice $\mathbf{0}$ : Zone où le $\mathrm{pH}$ n'a aucune influence sur l'oxydation et l'altération des minéraux. Le $\mathrm{pH}$ peut importer négativement sur la prolifération du DMA vis-à-vis de la présence des carbonates.

Indice 1 : Zone où le $\mathrm{pH} \sim 7$, zone normale, l'eau est proche de la neutralité. Ces zones sont considérées non ou légèrement touchées par le DMA. Elles renferment des eaux dont le pH est compris entre 6.5 et 7.5.

Indice 2 : Zone à pH entre 6,5 à 5.5, où la pyrite mise en contact avec cette eau, subissant ainsi, en premier lieu une oxydation chimique lente dite oxydation directe (Price, 2009). C'est là où le risque est très faible:

$$
2 \mathrm{FeS}_{2}+7 \mathrm{O}_{2}+2 \mathrm{H}_{2} \mathrm{O}===>2 \mathrm{Fe}^{2+}+4 \mathrm{SO}_{4}{ }^{2-}+4 \mathrm{H}^{+} \quad \text { (E3) }
$$

Indice 3: Zone où le milieu s'acidifie graduellement autour des sulfures, ensuite la deuxième étape du processus s'amorce. Cette étape est marquée par l'oxydation du fer ferreux en fer ferrique. C'est le champ correspondant à la précipitation d'un hydroxyde de fer $\mathrm{Fe}(\mathrm{OH})_{3}$ et la prolifération des protons $\mathrm{H}^{+}$. Cette précipitation contribuera à acidifier 
davantage le milieu, en engendrant ainsi, un $\mathrm{pH}$ de plus en plus bas suivant la réaction $(\mathrm{E} 4)$ :

$$
2 \mathrm{Fe}^{2+}+\mathrm{O}_{2}+2 \mathrm{H}^{+}===>2 \mathrm{Fe}^{3+}+2 \mathrm{OH}^{-}
$$

Il y a précipitation d'un hydroxyde de fer $\left(\mathrm{Fe}(\mathrm{OH})_{3}\right)$ (Miramond, 2006), selon la réaction suivante: $\quad \mathrm{Fe} 3++3 \mathrm{H} 2 \mathrm{O} \rightarrow \mathrm{Fe}(\mathrm{OH}) 3+3 \mathrm{H}+$ (E5)

Indice 4 : Zone, où le $\mathrm{pH}$ en deçà de 3,5 environ, le fer ferrique reste en solution et devient un agent oxydant, pouvant oxyder la pyrite, même en l'absence d'oxygène par voie indirecte (Morin \& Hutt, 1994). Cette réaction marque le phénomène d'auto-entretien de la génération du DMA, par l'établissement d'un processus cyclique mettant en jeu la réaction suivante:

$$
\mathrm{FeS}_{2}+14 \mathrm{Fe}^{3+}+8 \mathrm{H}_{2} \mathrm{O}===>15 \mathrm{Fe}^{2+}+2 \mathrm{SO}_{4}{ }^{2-}+16 \mathrm{H}^{+}
$$

Cette zone est considérée la plus génératrice du DMA. Elle correspond aux limites, là où l'ensemble de ces processus seraient catalysés par diverses bactéries, telles les bactéries thiobacillus ferrooxidan qui interviendraient directement en contact avec les sulfures (Toniazzo, 1998). Plusieurs minéraux primaires et même de néoformations peuvent contenir des métaux en concentrations importantes ou en traces, lesquels pourront passer en solution lors de la dissolution des minéraux (à faible $\mathrm{pH}$ ) et pourront aussi affecter la composition du DMA.

\section{III.2.6. Carte de la répartition de la conductivité électrique}

La conductivité électrique est évaluée par la capacité de la solution aqueuse à conduire le courant électrique. Elle dépend strictement de la concentration des différentes espèces ioniques en solution. Les mesures peuvent donner une estimation de la charge en ions dans le liquide. Elle est considérée comme indicateur de convergence des réactions d'oxydations actives. Le contraste est nettement marqué par la déférence de valeurs mesurées dans les concessions minières. Elles sont supérieures à proximité des points d'entreposage des rejets et plus faibles qu'on s'éloigne de ces points. Dans les zones à fortes valeurs, la minéralisation est très élevée et les réactions sont plus rapides, là où le phénomène de DMA s'est y intensifié. Outre, la conductivité électrique est liée conjointement de l'évolution de la répartition spatiale des rejets et plus proportionnellement rattachée à la variation des teneurs en matériaux sulfurés. Les cartes sont élaborées à base des mesures effectuées in situ, sur les eaux de ruissellement, les eaux stagnantes, les eaux souterraines à travers les puits et les galeries. Ces mesures sont prélevées en différentes périodes de l'année.

Le classement des zones a été établi à base des seuils de manière à obtenir un classement pertinent. Le principe de classement des zones en fonction de la qualité de la conductivité électrique a été établi comme suit: 
Indice 0 : Zone à très faible minéralisation, dont la conductivité électrique est strictement inférieure à $200 \mu \mathrm{S} / \mathrm{cm}$.

Indice 1 : Zone à faible minéralisation, dont la conductivité électrique est limitée comme suite : $200 \mu \mathrm{S} / \mathrm{cm}<$ Conductivité $<450 \mu \mathrm{S} / \mathrm{cm}$.

Indice 2 : Zone à moyenne minéralisation, dont la conductivité électrique est limitée est comme suite : $450 \mu \mathrm{S} / \mathrm{cm}<$ Conductivité < $1200 \mu \mathrm{S} / \mathrm{cm}$.

Indice 3 : Zone à minéralisation élevée, dont la conductivité électrique est limitée comme suite: $1200 \mu \mathrm{S} / \mathrm{cm}<$ Conductivité $<4400 \mu \mathrm{S} / \mathrm{cm}$.

Indice 4 : Zone à minéralisation très élevée, dont la conductivité électrique est strictement supérieure à $4400 \mu \mathrm{S} / \mathrm{cm}$

Ces valeurs ont été traitées vis-à-vis du degré de contamination incorporé comme qualité de minéralisation. La valeur $450 \mu \mathrm{S} / \mathrm{cm}$ est la valeur mesurée dans le puits $\mathrm{Lq}_{10}$ sis à Douar Ichamlalene au sud du Douar Ouled Haddou Rahhou. Ce puits a été source d'approvisionnement en eau potable au profit de la population locale. Pour la valeur $1200 \mu \mathrm{S} / \mathrm{cm}$, c'est la valeur limite admissible pour produire de l'eau potable selon la norme marocaine (N.M 03.7.001), et la valeur $4400 \mu \mathrm{S} / \mathrm{cm}$, correspond à la valeur mesurée dans l'un des puits abandonnés de Sétolazar. Les résultats de ce classement sont représentés dans la carte illustrée dans la figure 7 . Elle présente cinq zones définies conformément aux instructions précitées. Ces zones sont codifiées en indices pour faciliter leur superposition avec les autres cartes.

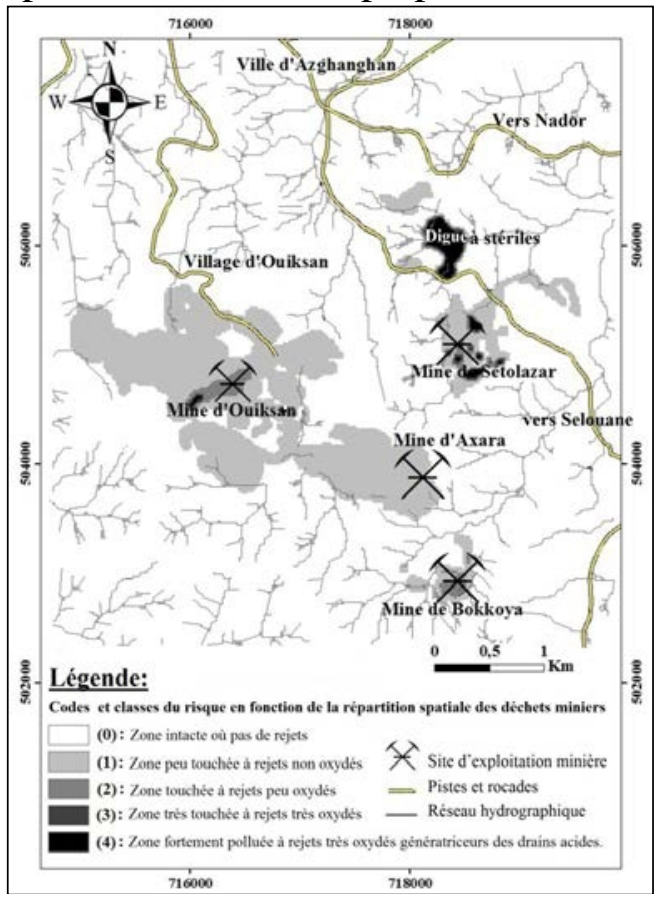

Fig. 2: Répartition qualitative des rejets miniers en fonction des degrés d'oxydation. 


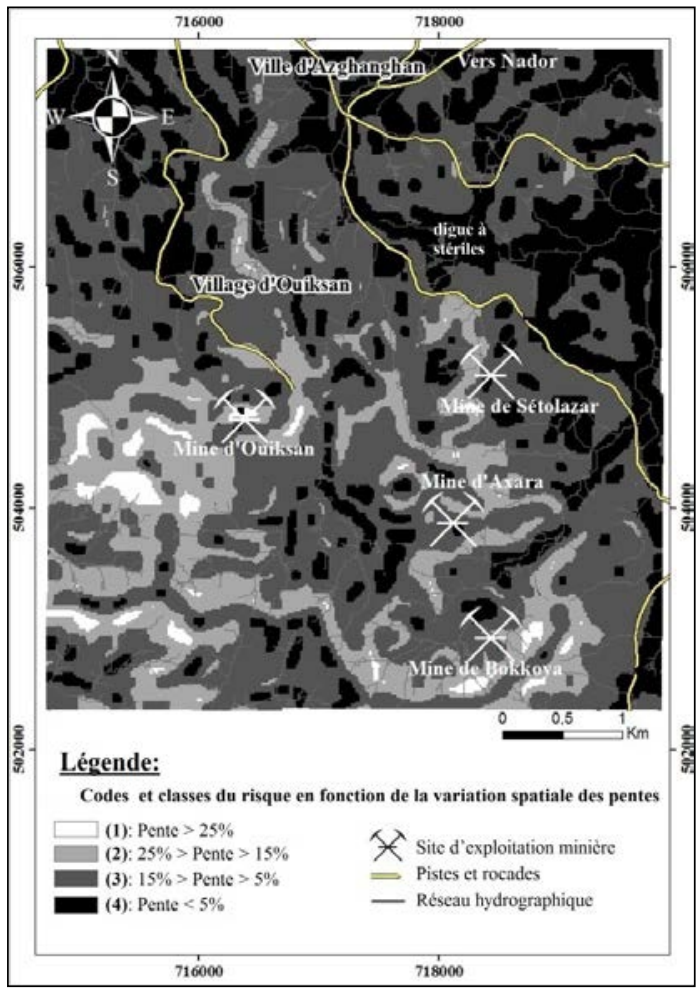

Fig. 3: Répartition qualitative des pentes en fonction de la susceptibilité de génération du DMA.

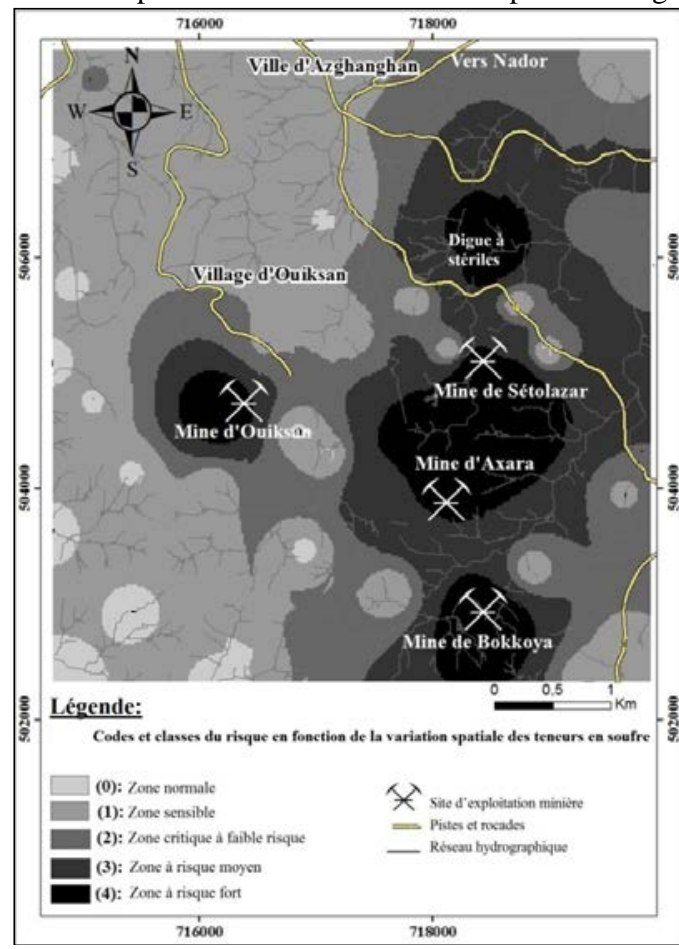

Fig. 4: Répartition quantitative des teneurs en soufre en fonction des degrés de contamination. 


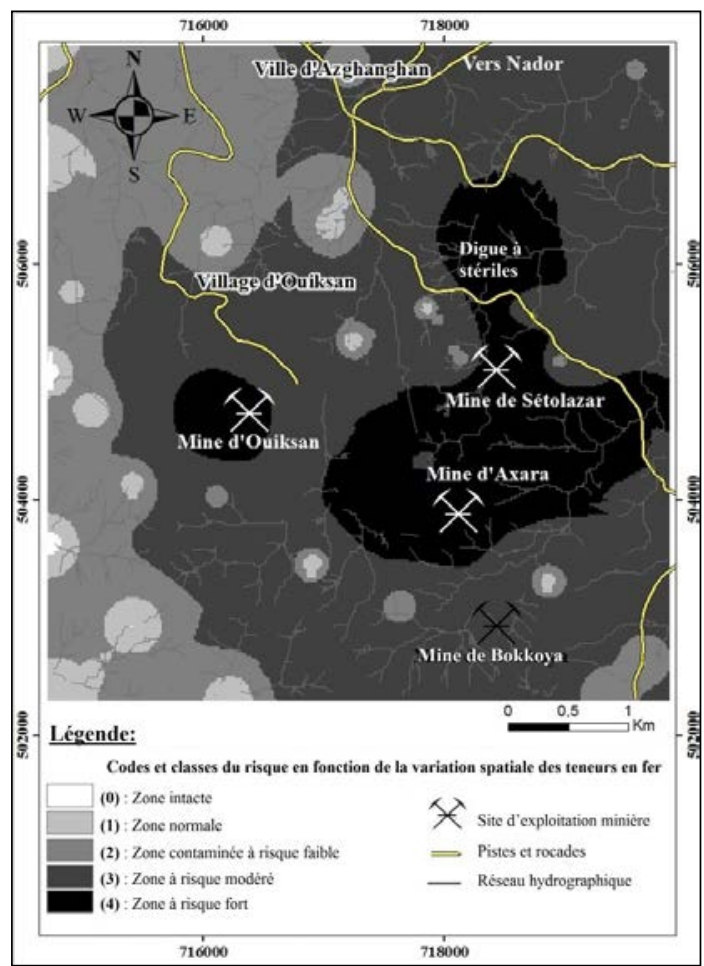

Fig. 5: Répartition quantitative des teneurs en fer en fonction des degrés de contamination.

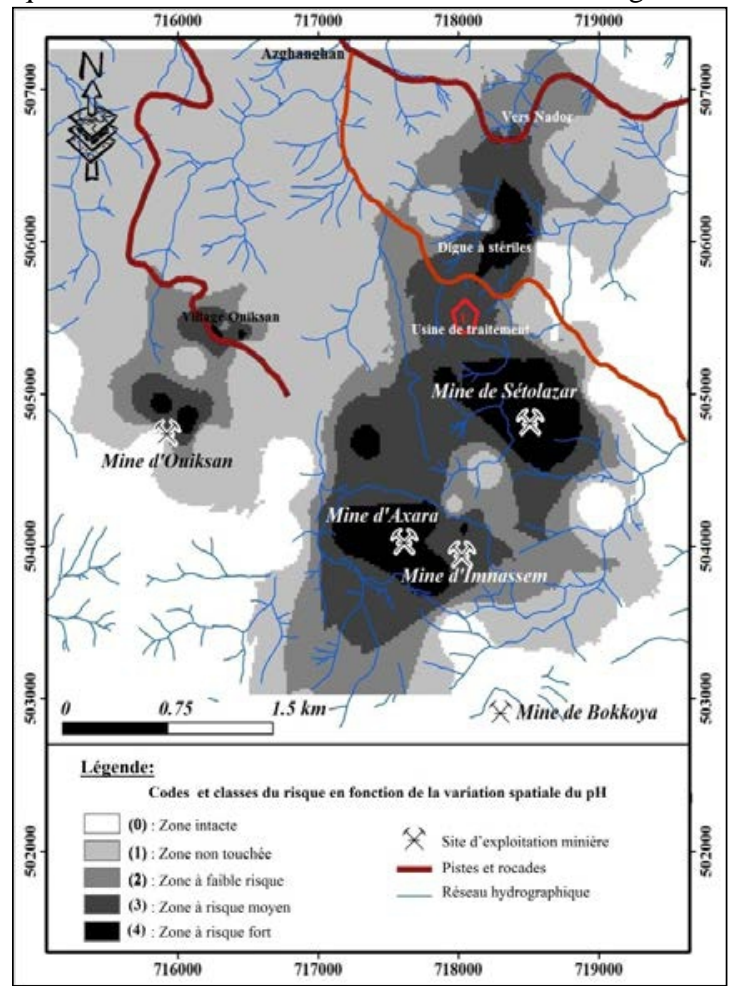

Fig. 6: Répartition d'acidité des milieux en fonction des degrés de contaminations. 


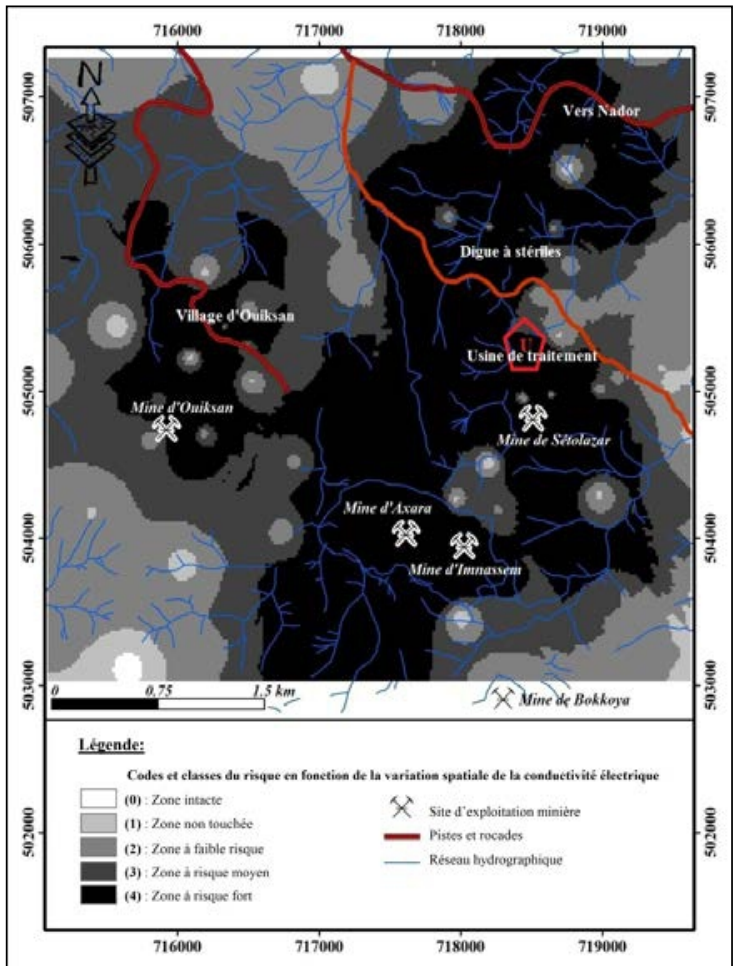

Fig. 7: Répartition de la minéralisation des liquides en fonction des degrés de contamination.

\section{III.3. Superposition des couches thématiques et différentiation des zones à risques}

Le degré de vulnérabilité dans l’espace étudié est défini ici comme l'intensité des contaminations, générées par des processus physico-chimiques complexes, résultant de la combinaison d'un ensemble de facteurs responsables de la production du DMA et la dégradation de l'environnement.

Les indicateurs de dégradation de l'environnement sont choisis vis-àvis de leurs importances dans l'accélération ou la restriction des processus de génération du DMA. Ces paramètres sont réunis pour mesurer le degré de dégradation des compartiments environnementaux, à titre d'exemple; les variations des teneurs en fer et en soufre contenues dans les solutions des solides ou dans les eaux ayant tellement des effets limitants sur la cinétique des réactions d'oxydation. Aussi, la qualité de la minéralisation, la nature des pentes et le degré d'acidité peuvent influencer largement les mécanismes de production de DMA, le relargage des métaux toxiques et la fragilisation des compartiments environnementaux.

Pour bien apprécier le degré de vulnérabilité dans la zone et perfectionner le choix des zones les plus prioritaires pour un aménagement prévu, les catégories qualitatives conclues de la superposition des cartes thématiques sont ainsi utilisées. Les classes des cartes thématiques comme 
déjà évoquées sont définies à base d’un raisonnement écologique, liée aux normes standards, en prenant en considérations les propriétés intrinsèques de la zone. Les cartes thématiques sont converties en mode «raster» et chaque unité pixel renferme une valeur comprise entre 0 et 4 . L'opération de la superposition des couches nous a permis d'additionner les valeurs des pixels qui caractérisent un point unique donné sur le site. Le résultat obtenu représente le degré de fragilité environnementale révélé. La solution de cette opération nous a permis ainsi d'établir la carte de la vulnérabilité environnementale, résultante d'assemblage des paramètres prédéfinis en valeurs dans les six couches thématiques (Fig. 8).

Cette carte a permis de déterminer cinq classes contrastées en fonction du degré de fragilisation, dans laquelle on peut différentier la présence effective des zones relativement intactes et d'autres, profondément contaminées par le drainage minier acide (DMA), en fonction des niveaux de contamination :

Zone intacte, corresponde généralement aux zones d'altitudes importantes et loin des points d'exploitation directe avec parfois des substratums carbonatés;

Zone non ou légèrement touchée par le DMA, corresponde aux zones plus ou moins écartées des points d'exploitation directe dont le substratum est carbonaté ;

Zone faiblement touchée par le DMA, corresponde aux zones situées dans le sens du drainage des écoulements provenus des zones touchées par le DMA ;

Zone touchée, corresponde aux zones à proximité des points d'entreposage et de stockage des rejets miniers solides, situées dans le sens de débordement et des afflux métallifères;

Zone fortement touchée, corresponde aux zones d'entreposage des déchets miniers très oxydés. Elle corresponde notamment aux points de dépôt des terrils et des tailings. 


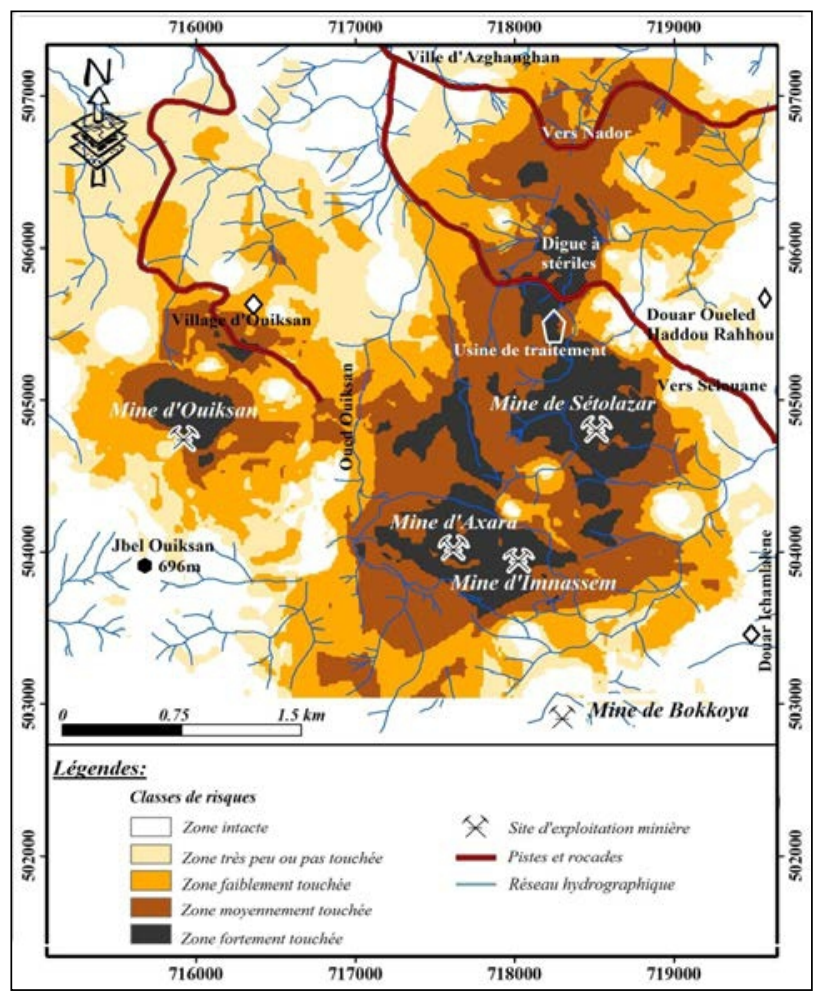

Fig. 8: Carte de la répartition de la vulnérabilité environnementale

Les zones intactes ou bien zones faiblement touchées sont localisées écartées des points d'exploitations directes et des aires de stockages des déchets miniers. Elles sont aperçues relativement protégées des drains acides par les altitudes et parfois neutralisés spontanément par les formations carbonatées des substratums (Lakrim et al, 2011). Les terrains les plus influencés, sont généralement de faibles pentes (>5\%), en forme de cuvettes, représentées souvent par des carrières d'excavations profondes où se rassemblent les eaux pluviales et les eaux de la nappe combinées de débris sulfurés très oxydés. Les surfaces des haldes à stériles, du bassin de rétention des résidus d'hydrométallurgie (tailings) et les alonteurs aussi, peuvent dévoiler une contamination approfondie par le DMA.

Les contaminations les plus importantes sont localisées notamment dans les carrières d'Axara, les galeries d'Ouiksan, les dépôts des tailings stockés à proximité de l'usine de traitement et au niveau de la digue à stériles de Sétolazar. Dans ces sites, l'oxydation est aperçue plus intensifiée, dont le $\mathrm{pH}$ des eaux est inférieur à 3 . Dans cet endroit, la teneur en fer mesuré dans un drain acide à Sétolazar est de 2,88 \%, tandis que le soufre est de 7,78\%. Ces sites sont excessivement chargés en éléments métalliques, avec des dépôts des oxydes, des hydroxydes de fer et des sulfates comme minéraux secondaires de néoformations (Fig. 9). 
Ces minéraux de néoformation s’y forment durant la période sèche, ils sont favorisés par la succession des déshydratations sous l'effet de la température, se précipitent, ainsi font stocker les métaux sous forme de substituants fragiles. Par la suite, et étant solubles, ces minéraux perdent leurs contenus en éléments métalliques pendant la saison humide. Par conséquent, ce cycle engendre des variations de concentrations en métaux dans le sol et dans la nappe phréatique (Lakrim et al, 2011). Le cycle est pérenne en absence des initiatives d'aménagement.
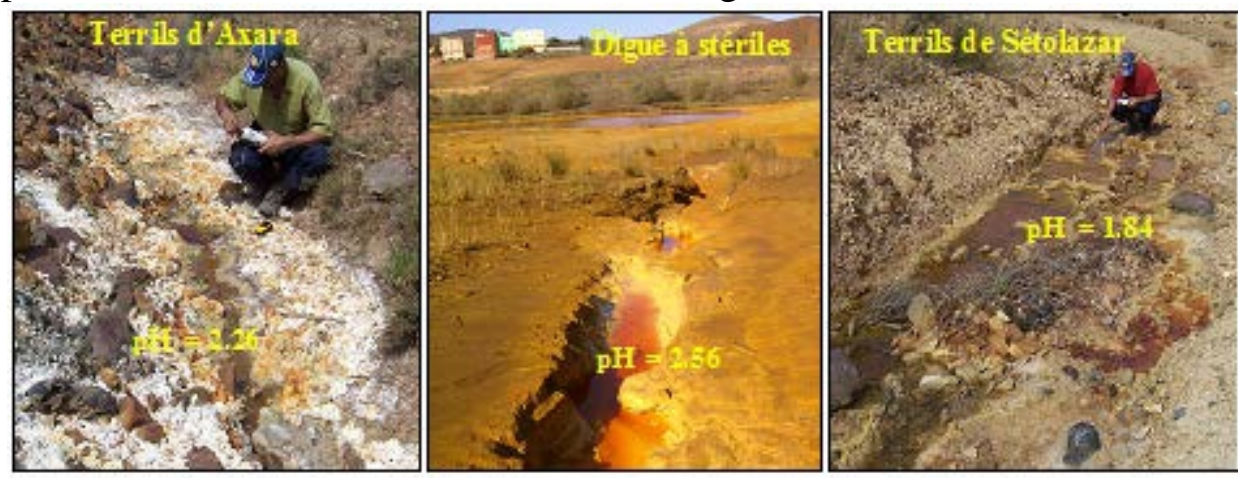

Fig. 9 : Photos illustrent des affleurements en minéraux de néoformation et des précipités d'oxydes, d'hydroxydes et des sulfates de fer (prises 2012).

\section{Conclusion}

La dégradation de la qualité environnementale dans la région minière est persiste, tandis que les principaux écosystèmes qui sous-tendent, entretiennent les ressources en eau et sols, continuent d'être fragilisés. Les défis de la pollution et la pression sur les ressources en eau qui en découle sont reconnus dorénavant comme un problème majeur. Bien que menées essentielle par la prolifération des réactions d'oxydation, en présence des stocks énormes de rejets miniers sulfurés, de faible granulométrie, exposés directement aux agents atmosphériques capables d'accélérer les processus de la pollution. Cette contamination est exacerbée par une évolution rapide du contexte environnemental qui comprend, entre autres, la dégradation de la nappe, la pénurie d'eau, la pollution des sols, la perte de la biodiversité et même peut contraindre la bonne conduite d'aménagement du grand projet de la fameuse lagune de Mar-Chica.

D'ailleurs, l'accent a été mis sur la caractérisation du comportement environnemental de ces déchets miniers. Les écosystèmes sont constatés très fragiles, l'acidité a pu enregistrer un $\mathrm{pH}$ de 0.87 . les puits de Sétolazar sont aperçus approfondément touchés et plus chargés en éléments métalliques, ils sont carrément abandonnés. Pareillement, le sol et le paysage sont apparus très dénaturés. La carte de vulnérabilité environnementale est considérée comme un fruit de la combinaison d'un ensemble de travaux scientifiques et 
techniques. Les prochaines études seront penchées sur l’aménagement et la gestion faisable de l'environnement minier de façon à valoriser l'ensemble des efforts déployés. Trois axes principaux peuvent être unis leurs contenus dans ce projet pour décrire la situation actuelle de la zone minière en parcourant l'enchainement des principales étapes. La première étape est déjà conclue dans les travaux antérieurs, c’est la caractérisation physique de la zone minière qui a visé à fournir une base de données convenable pour cartographier la vulnérabilité. La seconde étape a été consacrée aux évaluations des degrés de la vulnérabilité et le suivi. Enfin, la dernière étape sera dédiée à la modélisation spatio-temporelle de la vulnérabilité. Tous les détails abordés, les approches et les résultats d'analyses collectés, pourront être très utiles pour les futures initiatives d'assainissements.

\section{References:}

BenZaazoua, M., Bussiere, B., Kongolo, M., Mclaughlin, J., Marion, P., (2000): "Desulphurization feasibility of mine tailings for acid mine drainage control: study on four Canadian tailings". Inter. J. Miner. Process., 60: 5774.

Baize, D \& Terce, M., 2002: "Les éléments traces métalliques dans les sols : Approches fonctionnelles et spatiale". INRA, Paris, 2002, 561 p.

Chumbley, C.G \& Unwin, RJ., (2004): "Cadmium and lead content of vegetables crops grown on land with an history of sewage sludge application". Environmental Pollution2004, Series B, 4: 231-237.

El Mansouri. A. (1985): "Rapport de Synthèses géologiques et évaluation des réserves de la SEFFERIF (Nador)".p 3-7 et p 44-55. 1-09-1985.

Kaniki. T. A. (2008): "Caractérisation Environnementale des Rejets MineroMétallurgiques du Copperbelt Congolais". Thèse de Ph. D Sciences de l’Ingénieur, Université de Liège, Faculté des Sciences Appliquées 2008.

Lakrim. M., Mesrar. L., El-Arousi. O., Lahrach. A., El Garouani. À., Ben Abidate. L., Tabyaoui. H.. Chaouni A. et Jabrane R, (2011): "Etude d'impact des déchets miniers de la mine de Nador sur l'environnement (Nord-Est du Maroc)". Revue LJJE de l'ENSH, 2011, 18, p.76. www.ensh.dz Lakrim. M., Mesrar. L., El-Arousi. O. et Jabrane R, (2012): " Etude d'impact des déchets miniers de la mine ferrifère de nador sur l'environnement (Nord-Est du Maroc)". Revue Notes et Mémoires du Service Géologique N575 2012 pp152- 155.

Lakrim. M., Mesrar. L., El-Arousi. O. et Jabrane. R, (2012): "Localisation des zones vulnérables à la contamination par le DMA, moyennant le SIG: Cas de la mine de fer de Nador (Maroc nord oriental)". Revue des études appliquées en sciences de la terre, géographie et aménagement N8-2012 pp15-25 
Lakrim. M. (2015): "la pollution de l'environnement par le drainage minier acide généré par les déchets miniers de la mine ferrifère de Nador". Thèse de $\mathrm{Ph}$. D, Sciences et techniques de l’Ingénieur, Université Sidi Mohamed Ben Abdellah de Fès, Faculté des Sciences et Techniques. pp 251.

Margaa, K., 1994: "Essai de cartographie des risques naturels application à l'aménagement de la région d'Al Hociema (Rif, Nord Maroc)". Thèse de doctorat, Université de France-Comté Besanàon. 1994 196p.

Mendoza. G. A \& Macoun. P. (2000): "Application de l'analyse multicritère à l'évaluation des critères et indicateurs". Centre de Coopération Internationale en Recherche Agronomique pour le Développement (CIRAD), 34398, Montpellier Cedex 5, France, 84 p.

Miramond. N., Miau D., Brochard F., (2006) : "Diagnostic du phénomène Drainage Minier Acide sur des mines d'or primaire en Guyane française Evaluation des risques associés". 03 ${ }^{\text {Th }}$ Rapport 2006 GEM Impact DIREN. Guyane Française.2006. pp119.

Morin. K. A \& Hutt. N.M. (1994): "compliance and environmental protection". Prediction of water chemistry in open pits during operation and after closure Proceedings of the eighteenth annual British Columbia reclamation symposium; Mine reclamation.

PRICE. A.W. (2009): "Prediction Manual for Drainage Chemistry from Sulphidic Geologic Materials". MEND Report 1.20.1, pp 579, CANMET Mining and Mineral Sci. Lab. Smithers, British Columbia VOJ 2N. Chapitre 7. p 4-14.

Renaud. J. (2006): "De l'optimisation multicritère à l'aide à la décision : propositions de modèles de choix des recommandations à l'industriel lors de la conception et de la fabrication de produits nouveaux", 14e Atelier de Raiso Besançon, France.2006.

Toniazzo. V. (1998): "Approche des mécanismes d'oxydation de la pyrite par Thiobacillus ferrooxidans grâce à la modélisation morpho-chimique de la surface minérale: rôle fondamental des phases superficielles oxydées". Université Henri-Poincaré de Nancy. 1998. 